SELECCIONES MATEMÁTICAS
Universidad Nacional de Trujillo
ISSN: $2411-1783$ (Online)
Vol. 06(01): $31-34(2019)$

\title{
Bessel functions and the wave equation.
}

\section{Las funciones de Bessel y la ecuación de las ondas.}

\section{Alberto Torchinsky*}

Received, Jan. 15, 2019

Accepted, Mar. 12, 2019

DOI: http://dx.doi.org/10.17268/sel.mat.2019.01.05

\begin{abstract}
We solve the Cauchy problem for the n-dimensional wave equation using elementary properties of the Bessel functions.
\end{abstract}

Keywords. Bessel functions, Wave Equation.

\section{Resumen}

Resolvemos el problema de Cauchy para la ecuación de las ondas en n dimensiones utilizando propiedades elementales de las funciones de Bessel.

Palabras clave. Funciones de Bessel, Ecuación de las Ondas.

1. Introduction. With $\nabla^{2}=D_{x_{1} x_{1}}^{2}+\cdots+D_{x_{n} x_{n}}^{2}$ the Laplacian in $\mathbb{R}^{n}$, where

$$
D_{x_{k} x_{k}}^{2}=\frac{\partial^{2}}{\partial x_{k}^{2}}, \quad 1 \leq k \leq n,
$$

and $D_{t}$ and $D_{t t}$ indicating the first and second order derivatives with respect to the variable $t \in \mathbb{R}$, respectively, the wave equation in the upper half-space $\mathbb{R}_{+}^{n+1}$ is given by

$$
D_{t t}^{2} u(x, t)=\nabla^{2} u(x, t), \quad x \in \mathbb{R}^{n}, t>0,
$$

and the Cauchy problem for this equation consists of finding $u(x, t)$ that satisfies (1.1) subject to the initial conditions

$$
u(x, 0)=\varphi(x) \quad \text { and } \quad D_{t} u(x, 0)=\psi(x), \quad x \in \mathbb{R}^{n},
$$

where for simplicity we shall take $\varphi$ and $\psi$ in $\mathcal{S}\left(\mathbb{R}^{n}\right)$. Applying the Fourier transform to (1.1) in the space variables, considering $t$ as a parameter, it readily follows that $\widehat{\nabla^{2}} u(\xi, t)=-|\xi|^{2} \widehat{u}(\xi, t)$, and so $\widehat{u}$ satisfies

$$
D_{t t}^{2} \widehat{u}(\xi, t)+|\xi|^{2} \widehat{u}(\xi, t)=0, \quad \xi \in \mathbb{R}^{n}, t>0
$$

subject to

$$
\widehat{u}(\xi, 0)=\widehat{\varphi}(\xi) \quad \text { and } \quad D_{t} \widehat{u}(\xi, 0)=\widehat{\psi}(\xi), \quad \xi \in \mathbb{R}^{n}
$$

For each fixed $\xi \in \mathbb{R}^{n}$ this resulting ordinary differential equation in $t$ is the simple harmonic oscillator equation with constant angular frequency $|\xi|$, and so

$$
\widehat{u}(\xi, t)=\widehat{\varphi}(\xi) \cos (t|\xi|)+\widehat{\psi}(\xi) \frac{\sin (t|\xi|)}{|\xi|}, \quad \xi \in \mathbb{R}^{n}, t>0 .
$$

\footnotetext{
*Department of Mathematics, Indiana University, Bloomington, Indiana 47405, USA(torchins@indiana. edu). 
Hence, the Fourier inversion formula gives for $(x, t) \in \mathbb{R}_{+}^{n+1}$,

$$
\begin{aligned}
u(x, t)=\frac{1}{(2 \pi)^{n}} \int_{\mathbb{R}^{n}} \widehat{\varphi}(\xi) & \cos (t|\xi|) e^{i \xi \cdot x} d \xi \\
& +\frac{1}{(2 \pi)^{n}} \int_{\mathbb{R}^{n}} \widehat{\psi}(\xi) \frac{\sin (t|\xi|)}{|\xi|} e^{i \xi \cdot x} d \xi .
\end{aligned}
$$

Since the first integral in (1.2) can be obtained from the second by differentiating with respect to $t$, we will concentrate on the latter. The idea is to interpret $\sin (|\xi| t) /|\xi|$ as the Fourier transform of a tempered distribution, and the key ingredient for this are the representation formulas established in [1].

2. Representation Formulas. The reader will note that the formulas are different in character when the dimension is odd on the one hand and even on the other, this is not unusual in $n$-dimensional Fourier analysis.

Assume that $n$ is an odd integer greater than or equal to 3. Then, with $d \sigma$ the element of surface area on $\partial B(0, R)$

$$
\frac{\sin (R|\xi|)}{|\xi|}=c_{n}\left(\frac{1}{R} \frac{\partial}{\partial R}\right)^{(n-3) / 2}\left(\frac{1}{\omega_{n} R} \int_{\partial B(0, R)} e^{-i x \cdot \xi} d \sigma(x)\right)
$$

where $R>0, \omega_{n}$ is the surface measure of the unit ball in $\mathbb{R}^{n}$, and $c_{n}^{-1}=(n-2)(n-4) \cdots 1$.

On the other hand, if $n$ is an even integer greater than or equal to 2 ,

$$
\frac{\sin (R|\xi|)}{|\xi|}=d_{n}\left(\frac{1}{R} \frac{\partial}{\partial R}\right)^{(n-2) / 2}\left(\frac{1}{v_{n}} \int_{B(0, R)} \frac{1}{\sqrt{R^{2}-|x|^{2}}} e^{-i x \cdot \xi} d x\right)
$$

where $R>0, d_{n}^{-1}=n(n-2)(n-4) \cdots 2$, and $v_{n}$ is the volume of the unit ball in $\mathbb{R}^{n}$.

The purpose of this note is to establish (2.1) and (2.2) using elementary properties of Bessel functions. $J_{\nu}(x)$, the Bessel function of order $\nu$, is defined as the solution of the second order linear equation

$$
x^{2} \frac{d^{2} y}{d x^{2}}+x \frac{d y}{d x}+\left(x^{2}-\nu^{2}\right) y=0 .
$$

Several basic properties of the Bessel functions follow readily from their power series expression [2]. They include the recurrence formula

$$
\frac{d}{d x}\left(x^{\nu} J_{\nu}(x)\right)=x^{\nu} J_{\nu-1}(x)
$$

the integral representation of Poisson type

$$
J_{\nu}(x)=\frac{(x / 2)^{\nu}}{\Gamma(\nu+1 / 2) \Gamma(1 / 2)} \int_{-1}^{1}\left(1-s^{2}\right)^{\nu-1 / 2} e^{i x s} d s,
$$

and the identity

$$
J_{1 / 2}(x)=\frac{\sqrt{2}}{\sqrt{\pi}} \frac{1}{x^{1 / 2}} \sin (x)
$$

for $x>0$.

We will consider the odd dimensional case first. The dimensional constant $c_{n}$ may vary from appearance to appearance until it is finally determined at the end of the proof. To begin recall that for $n \geq 3$, as established in (18) in [1],

$$
\frac{1}{\omega_{n-1} R} \int_{\partial B(0, R)} e^{-i x \cdot \xi} d \sigma(x)=R^{n-2} \int_{-1}^{1} e^{i R|\xi| s}\left(1-s^{2}\right)^{(n-3) / 2} d s,
$$

which combined with (2.4) above with $\nu-1 / 2=(n-3) / 2$ there, i.e., $\nu=(n-2) / 2$, gives

$$
\begin{aligned}
\frac{1}{\omega_{n} R} \int_{\partial B(0, R)} e^{-i x \cdot \xi} d \sigma(x) & =c_{n} R^{n-2} \frac{J_{(n-2) / 2}(R|\xi|)}{(R|\xi|)^{(n-2) / 2}} \\
& =c_{n} \frac{1}{|\xi|^{n-2}}(R|\xi|)^{(n-2) / 2} J_{(n-2) / 2}(R|\xi|) .
\end{aligned}
$$


Now, by (2.3) we obtain that

$$
\frac{\partial}{\partial R}\left(\frac{1}{\omega_{n} R} \int_{\partial B(0, R)} e^{-i x \cdot \xi} d \sigma(x)\right)=c_{n} \frac{1}{|\xi|^{n-2}}|\xi|(R|\xi|)^{(n-2) / 2} J_{(n-4) / 2}(R|\xi|),
$$

or

$$
\frac{1}{R} \frac{\partial}{\partial R}\left(\frac{1}{\omega_{n} R} \int_{\partial B(0, R)} e^{-i x \cdot \xi} d \sigma(x)\right)=c_{n} \frac{1}{|\xi|^{n-4}}(R|\xi|)^{(n-4) / 2} J_{(n-4) / 2}(R|\xi|) .
$$

Thus, applying the above reasoning $(n-3) / 2$ times, (2.5) gives

$$
\begin{aligned}
\left(\frac{1}{R} \frac{\partial}{\partial R}\right)^{(n-3) / 2}\left(\frac{1}{\omega_{n} R}\right. & \left.\int_{\partial B(0, R)} e^{-i x \cdot \xi} d \sigma(x)\right) \\
& =c_{n} \frac{1}{|\xi|}(R|\xi|)^{1 / 2} J_{1 / 2}(R|\xi|) \\
& =c_{n} \frac{1}{|\xi|}(R|\xi|)^{1 / 2} \frac{\sin (R|\xi|)}{(R|\xi|)^{1 / 2}} \\
& =c_{n} \frac{\sin (R|\xi|)}{|\xi|} .
\end{aligned}
$$

The value of $c_{n}$ is readily obtained as in [1], and (2.1) has been established.

To consider the case $n$ even, one generally proceeds at this point by a reasoning akin to Hadamard's method of descent, i.e., the desired result for the wave equation in even dimension $n$ is derived from the result in odd dimension $n+1$, as is done for instance in [1] for the representation formulas. On the other hand, Bessel functions provide the desired result for the wave equation in even dimensions directly, by a method akin to ascent: the result for the wave equation for dimension $n=2$ is obtained explicitly, and for even dimension $n+2$ is obtained from the result in even dimension $n$.

We will first prove a preliminary result. The dimensional constant $d_{n}$ may vary from appearance to appearance until it is finally determined at the end of the proof.

Lemma 2.1. The following three statements hold.

$$
\int_{0}^{\infty} \sin (R \rho) J_{0}(t \rho) d \rho=\frac{1}{\sqrt{R^{2}-t^{2}}} H(R-t), \quad R, t>0,
$$

where $H$ denotes the Heavyside function.

Furthermore, for $\nu \geq 1$,

$$
\left(\frac{1}{R} \frac{\partial}{\partial R}\right)\left(\int_{0}^{\infty} \sin (R \rho) \rho^{\nu-1} J_{\nu-1}(t \rho) d \rho\right)=\frac{1}{t} \int_{0}^{\infty} \sin (R \rho) \rho^{\nu} J_{\nu}(t \rho) d \rho,
$$

and, consequently, for $1 \leq j \leq \nu$,

$$
\left(\frac{1}{R} \frac{\partial}{\partial R}\right)^{j}\left(\int_{0}^{\infty} \sin (R \rho) \rho^{\nu-j} J_{\nu-j}(t \rho) d \rho\right)=\frac{1}{t^{j}} \int_{0}^{\infty} \sin (R \rho) \rho^{\nu} J_{\nu}(t \rho) d \rho .
$$

Proof. (2.6) is Formula (6) in [2], page 405.

Now,

$$
\begin{aligned}
\frac{\partial}{\partial R}\left(\int_{0}^{\infty} \sin (R \rho) \rho^{\nu-1} J_{\nu-1}(t \rho) d \rho\right) & =\int_{0}^{\infty} \cos (R \rho) \rho^{\nu} J_{\nu-1}(t \rho) d \rho \\
& =\frac{1}{t^{\nu}} \int_{0}^{\infty} \cos (R \rho)(t \rho)^{\nu} J_{\nu-1}(t \rho) d \rho
\end{aligned}
$$

which, by (2.3), equals

$$
\frac{1}{t^{\nu+1}} \int_{0}^{\infty} \cos (R \rho) \frac{\partial}{\partial \rho}\left((t \rho)^{\nu} J_{\nu}(t \rho)\right) d \rho=R \frac{1}{t} \int_{0}^{\infty} \sin (R \rho) \rho^{\nu} J_{\nu}(t \rho) d \rho,
$$

which proves (2.7).

(2.8) follows by repeated applications of (2.7), and we have finished. $\square$

Finally, recall that the Fourier transform of a radial function $f$ on $\mathbb{R}^{n}$ is given by the expression [2],

$$
\widehat{f}(|\xi|)=d_{n} \frac{1}{|\xi|^{(n-2) / 2}} \int_{0}^{\infty} \rho^{n / 2} f(\rho) J_{(n-2) / 2}(|\xi| \rho) d \rho .
$$


In particular, we have

$$
\int_{\mathbb{R}^{n}} \frac{\sin (R|\xi|)}{|\xi|} e^{-i x \cdot \xi} d \xi=d_{n} \frac{1}{|x|^{(n-2) / 2}} \int_{0}^{\infty} \rho^{n / 2} \frac{\sin (R \rho)}{\rho} J_{(n-2) / 2}(|x| \rho) d \rho .
$$

Let now $n=2 k$ be an even integer. Then by (2.9),

$$
\int_{\mathbb{R}^{n}} \frac{\sin (R|\xi|)}{|\xi|} e^{-i x \cdot \xi} d \xi=d_{n} \frac{1}{|x|^{(k-1)}} \int_{0}^{\infty} \sin (R \rho) \rho^{k-1} J_{k-1}(|x| \rho) d \rho
$$

and, therefore, (2.8) with $\nu=j=k-1$ there yields

$$
\begin{aligned}
\int_{\mathbb{R}^{n}} \frac{\sin (R|\xi|)}{|\xi|} e^{-i x \cdot \xi} d \xi & =d_{n} \frac{1}{|x|^{(k-1)}} \int_{0}^{\infty} \sin (R \rho) \rho^{(k-1)} J_{k-1}(|x| \rho) d \rho \\
& =d_{n}\left(\frac{1}{R} \frac{\partial}{\partial R}\right)^{(k-1)}\left(\int_{0}^{\infty} \sin (R \rho) J_{0}(|x| \rho) d \rho\right) \\
& =d_{n}\left(\frac{1}{R} \frac{\partial}{\partial R}\right)^{(k-1)}\left(\frac{1}{\sqrt{R^{2}-|x|^{2}}} H(R-|x|)\right) .
\end{aligned}
$$

Thus by the Fourier inversion formula,

$$
\begin{aligned}
\frac{\sin (R|\xi|)}{|\xi|} & =d_{n}\left(\frac{1}{R} \frac{\partial}{\partial R}\right)^{(n-2) / 2}\left(\int_{\mathbb{R}^{n}} \frac{1}{\sqrt{R^{2}-|x|^{2}}} H(R-|x|) e^{-i x \cdot \xi} d x\right) \\
& =d_{n}\left(\frac{1}{R} \frac{\partial}{\partial R}\right)^{(n-2) / 2}\left(\frac{1}{v_{n}} \int_{B(0, R)} \frac{1}{\sqrt{R^{2}-|x|^{2}}} e^{-i x \cdot \xi} d x\right)
\end{aligned}
$$

The constant $d_{n}$ is readily determined as in [1], and we have finished.

\section{REFERENCES}

[1] Torchinsky, A., The Fourier transform and the wave equation, Amer. Math. Monthly 118(7) (2011), 599-609.

[2] Watson, G. N., A treatise on the theory of Bessel functions. Cambridge Mathematical Library. Cambridge University Press, Cambridge, 1995. 